\title{
Élites transnationales
}

Introduction

le Comité de rédaction

\section{(2) OpenEdition \\ Journals}

Édition électronique

URL : http://journals.openedition.org/conflits/18986

DOI : $10.4000 /$ conflits. 18986

ISSN : $1777-5345$

Éditeur :

CECLS - Centre d'études sur les conflits - Liberté et sécurité, L'Harmattan

Édition imprimée

Date de publication : 15 octobre 2015

Pagination : 7-13

ISBN : 978-2-343-07829-8

ISSN : 1157-996X

Référence électronique

le Comité de rédaction, «Élites transnationales », Cultures \& Conflits [En ligne], 98 | été 2015, mis en ligne le 15 octobre 2015, consulté le 02 avril 2021. URL : http://journals.openedition.org/conflits/ 18986 ; DOI : https://doi.org/10.4000/conflits.18986 


\title{
Elites transnationales
}

\author{
Introduction
}

\section{Par le Comité de rédaction de Cultures \& Conflits}

e numéro de Cultures E Conflits est issu d'une table ronde organisée
dans le cadre du 12ème congrès de l'Association Française de Science Politique (AFSP) en juillet 2013. Cette rencontre visait à réunir des chercheur(e)s se réclamant d'une sociologie politique de l'international au sein de laquelle la sociologie critique, puisant ses racines dans les travaux de Norbert Elias et de Pierre Bourdieu, occupe une place de premier rang. Dans une telle perspective scientifique, l'anthropologie politique, soucieuse du terrain et des savoirs réflexifs des acteurs d'un univers social donné, est centrale.

Il ne s'agit donc pas de renouer avec le fonctionnalisme et les approches d'une science politique américanisée, pour qui la sociologie politique de l'international serait un sous-domaine des études de politique internationale permettant simplement de préciser des grandes hypothèses théoriques en mettant un peu de chair sur les acteurs désincarnés qui hantent leurs récits. Le projet d'une sociologie politique de l'international n'est en effet pas de donner de l'épaisseur (thickness) socio-historique aux récits traditionnels concernant les États, les organisations internationales ni de mieux repérer les systèmes d'action et les mécanismes d'intégration. Ceux qui se réclament de cette perspective se différencient en ce sens des tenants d'une English school attentive à la sociologie historique et que Barry Buzan continue d'appeler de ses vœux.

Il s'agit, avec le développement au sein de l'International Studies Association (ISA) de la section International Political Sociology - IPS (ainsi que la revue du même nom) et de son pendant français (fortement porté par les réflexions menées dans la revue Cultures $\mathcal{E}$ Conflits) de révéler les différents impensés et formes de sens commun qui se présentent comme des savoirs disciplinaires alors que très souvent il ne s'agit que d'orthodoxies d'un moment, de dogmes qui se prétendent méthodes. 
Les perspective mises en avant dans IPS invitent les chercheur(e)s à être résolument " hétérodoxes », à mettre en tension les raisonnements logiques, les récits et les positions de pouvoir qui ont fait jusqu'à maintenant que les Relations Internationales (RI) aient pu se prétendre exceptionnelles en termes d'objet, de méthodes et d'épistèmé. Il est au contraire nécessaire de penser l'articulation des RI à une théorie politique prenant au sérieux ce que les relations internationales disent d'elles-mêmes et ce qui a empêché la théorie politique de questionner l'international, en se repliant sur le national-sociétal ou en sautant la question pour se déclarer globale. Il faut aussi penser leur articulation avec la géographie critique et les notions d'espaces pluriels qui remettent en cause nombre de réflexions sur le territoire. Enfin, et surtout, il faut être à même de penser le «pari » intellectuel qui vise à explorer les contradictions des savoirs disciplinaires, à en faire la socio-genèse et présenter, à partir de là, des formes alternatives de mise en relation des pratiques sociales.

Ces « lignes transversales » articulent le politique et les diverses formes de politisation, ainsi que la dimension anthropologique qui sous-tend le rapport à ce qu'on appelle l'observation sur le terrain et les modes de raisonnement sur l'humanité, la citoyenneté, le cosmopolite. Elles encouragent une démarche de recherche qui part des pratiques des acteurs et analyse leurs relations de distinction et de mimétisme, ainsi que les processus qui encadrent ces relations. Elles interrogent la question de l'international qui ne peut être réduite ni à une question de niveau d'analyse ou d'objet ayant une existence propre et des règles différentes, ni à un devenir global qui amènerait le monde contemporain à devenir une société mondiale sans frontières. Elles invitent à penser cet espace international en terme de dynamiques centrifuges des champs de pouvoir déstabilisant les cadres traditionnels des rapports d'attraction vers un centre de pouvoir caractérisant tant les États que les marchés. Elles amènent enfin à repenser le rapport entre cet international et la sociologie et à penser le social sans se référer immédiatement à une société civile capturée par un État, mais au contraire à une multiplicité des univers sociaux dans lesquels un monde pluriel est composé d'hommes pluriels.

Cette Paris School, comme elle a été nommée dans les cercles des internationalistes anglo-américains et qui est intimement liée à la revue Cultures $\mathcal{E}$ Conflits, désigne cette façon particulière d'approcher l'international. De nombreuses publications lui ont donné une forte visibilité. Pourtant, si les débats qui ont permis de constituer IPS au sein de l'ISA sont particulièrement riches en Angleterre, au Canada, au Brésil, ou encore dans les pays nordiques, ils n'ont pas eu le même impact en France, où les manuels de relations internationales continuent de présenter uniquement les façons les plus traditionnelles d'étudier l'interétatique et la «globalisation». 
Il était plus que temps que cela change et c'est le sens du pari de proposer systématiquement des sessions IPS dans les congrès de l'AFSP. Les deux tables rondes de 2013 ont permis de faire « retour » sur deux questions centrales qu'une démarche IPS peut éclairer différemment : (1) celle de l'analyse des positions dominantes dans un univers social donné et (2) celle de l'analyse des mécanismes de violence, de pouvoir symbolique, d'arbitraire au sein même des mécanismes dits de sécurisation ou d'(in)sécurisation.

La première question fait l'objet de ce numéro. La transdisciplinarité de la démarche IPS est un moyen de rediscuter les questions les plus centrales et les plus classiques de la science politique et des relations internationales avec d'autres instruments d'analyse, débouchant sur la remise en cause des terminologies les plus banales et les plus quotidiennes de la discipline. Elle permet de se mettre à distance des formes d'essentialisme, et souvent de pensée coloniale, qui caractérisent cette dernière.

Nous abordons donc ici cette question des «élites internationales ». Loin d'accepter l'idée d'une élite, d'une méritocratie émergeant de la diversité sociale, ou d'une oligarchie se reproduisant à travers des capitaux sociaux et familiaux, ce numéro interroge ce qui se joue dans la formation de positions dominantes qui se reproduisent d'une génération sur l'autre, et ce malgré des transformations profondes des enjeux internes de chaque univers social particulier. Comment circulent les mécanismes qui assurent certains modes de reproduction et comment sont-ils compartimentés, nationalement ou professionnellement ? Comment se traduisent des expériences socio-historiques différentes, mais aussi leurs points de passages obligés, et les dynamiques sociales de diffraction et d'expansion qui leur sont inhérentes ?

Nous ouvrons ce dossier par un long entretien avec Yves Dezalay, dans lequel il retrace les enquêtes qu'il a réalisées, seul ou avec Bryant Garth, sur ce qu'il appelle l'internationalisation des noblesses d'État et tout particulièrement sur le groupe des «lawyers». Il revient ainsi sur l'univers de ces «marchands de droit », sur les oppositions internes à ce champ, notamment entre professeurs européens et jeunes avocats d'affaire américains, et sur les modes de compréhension des mécanismes d'héritage, des dynamiques de transformation des positions et des recompositions de pratiques professionnelles a priori plus ou moins similaires mais fortement marquées par leur inscription dans des champs nationaux particuliers.

Comme Yves Dezalay le signale avec force, il ne s'agit pas de travailler a priori sur des catégories comme un « champ juridique » ou sur les «professionnels du droit ", mais sur les dynamiques à partir desquelles un petit groupe précis d'acteurs a une stratégie qui le place à la frontière entre l'héritage social, le champ du pouvoir d'État, les champs ou les espaces du pouvoir 
international, et le monde académique. C'est cette étude qui permet de comprendre les recompositions structurales de ces espaces interdépendants, leur socio-genèse et leur diffraction spatiale. La réflexion sur les relations entre les univers du droit, de la finance et de l'audit permet alors de comprendre comment la question dite de « l'arbitrage » relève de batailles territoriales et de rivalités de cousinage, ouvrant ainsi la voie à une question plus ambitieuse qui est celle d'une démarche sociologique plus attentive aux différences professionnelles qui sont à la fois le produit d'histoires nationales mais qui nourrissent aussi la compétition internationale. Il ressort de cet entretien la notion d' " agent double », ainsi qu'une réflexion sur les pratiques des juristes venant d'Inde, de Corée, du Brésil, et qui permettent de comprendre les « guerres de palais »dans lesquelles ils s'engagent. Ainsi Dezalay nous dit que ce qui compte sociologiquement, «ce n'est pas simplement qui sont ces gens-là, mais ce qu'ils contribuent à produire en termes d'institutions d'État, de dispositifs de régulation ». La question des frontières du champ dans lequel ils évoluent ne prend sens qu'en relation avec les effets pratiques qui y sont liés. À ce titre, Dezalay parle d' " une sorte d'obsolescence des technologies et du savoir juridiques qui est inscrite dans la dynamique structurale de ces champs professionnels, et qui peut être plus ou moins accélérée selon les moments » et ce serait elle, dans ce cas, qui permettrait de comprendre le cadrage des luttes et leurs dynamiques. Comme le lecteur attentif le remarquera, ce que Dezalay nous donne à voir dans cet entretien, au delà de la description de son travail et de sa démarche, c'est un retour réflexif sur les modalités d'enquêtes et d'observation qui part d'un refus de poser des cadres a priori et de s'enfermer dans des questions rhétoriques de définition et de techniques qui seraient les seules appropriées. Au contraire, pour lui, il est plus intéressant de maintenir l'hypothèse de champs, et de la faire fructifier au lieu de la refermer à peine posée comme si elle était un cadre d'analyse, un système, une structure. En effet l'hypothèse d'un champ vaut bien plus par sa valeur heuristique sur les dynamiques à l'œuvre et sur la possibilité de reformuler les questions de recherche que sur une quelconque valeur de causalité que certains chercheurs tendent à lui conférer en s'enfermant dans un certain déterminisme. Les questions de méthode sont alors performatives et non reproductibles à l'identique. Nous sommes loin de l'orthodoxie des manuels de méthode qui les veulent reproductibles. Les méthodes dépendent de l'imagination sociologique des chercheurs, et des trajectoires propres des enquêteurs, de leurs relations et capitaux spécifiques, des dynamiques historiques dont ils sont les témoins, et qui favorisent ou non, l'ouverture des univers sociaux à leurs questionnements. Les expériences de vie et de recherche, les conseils qui peuvent en sortir sont utiles, mais il ne peut s'agir d'un «catalogue de recettes pour "appliquer" » la théorie des champs à un " objet » particulier. Un anti-discours de la méthode se met en place à partir d'une leçon de vie et d'humilité joyeuse qui a aussi pour effet de contrer ceux qui veulent faire de Bourdieu un dogme amendable en fonction d'objectifs politiques ou une méthode d'analyse soluble dans la 
science politique classique. Il n'y a pas à douter que cet entretien au ton décontracté pose des questions centrales sur le type de sociologie que l'on compte entreprendre et sur les objectifs qui sont ceux du chercheur, et nous espérons qu'il donnera lieu à des commentaires et des réactions significatives.

Dans la même veine qu'Yves Dezalay, et sur la base de très nombreux entretiens, voyages et études de documents concernant les projets de développement et de sécurité associés aux frontières des pays d'Asie centrale, Médéric Martin-Mazé présente une recherche sur ce qu'il appelle l'extension transnationale du domaine de la lutte symbolique : comment les savoirs d'État sur les frontières passent-ils les frontières de l'État ? Se distanciant des approches neo-institutionalistes et constructivistes idéalistes qui lisent les actions des organisations comme des réponses à des menaces transnationales, il insiste sur le fait que les doctrines de gestion des frontières de l'Union européenne et de l'Organisation pour la sécurité et la coopération en Europe (OSCE) sont forgées et mises en circulation en Asie Centrale à l'aune de luttes qui ont pour enjeu générique l'imposition de la définition légitime de la réalité des menaces, et pour enjeu spécifique la monopolisation des moyens légitimes de circulation. À mesure que le monopole étatique de la violence symbolique se fragmente, des guildes professionnelles poursuivent alors leurs luttes dans un espace transnational qui tend à se densifier et à se différencier. Cela permet dès lors de repérer des scènes différentes où joue la différenciation entre les sièges des organisations et les terrains centrasiatiques, où s'opposent en fait gardesfrontières finlandais et austro-hongrois. À partir de cette opposition dont il retrace la genèse, il ressort une image différente de l'économie des luttes au sein de l'espace de sécurité intérieure européen et du rôle de l'agence Frontex, ainsi qu'une analyse précise des stratégies sur les différents terrains qui aboutissent à des chassés-croisés de position. Se référant à Bernard Lahire, il conclut en considérant que l'analyse des pratiques internationales doit se faire au croisement de contextes sociaux particuliers, produits d'un processus de différenciation socio-historique au long cours, et de patrimoines pluriels de dispositions, résultant de parcours de socialisation professionnelle multiples.

Avec une ambition plus formalisatrice s'appuyant sur les interlocking directorate studies et mobilisant les analyses de réseaux, un article co-écrit par Philippe Blanchard, François-Xavier Dudouet et Antoine Vion se propose de faire une analyse structurale et séquentielle des élites économiques transnationales. Selon eux, et s'inspirant aussi des travaux d'Yves Dezalay, «l'identification et la description fine des acteurs qui structurent cet espace transnational contribuent à mettre à jour le phénomène transnational lui-même ». Ils proposent ainsi de s'attacher aux dirigeants de grandes entreprises qui structurent le cœur du milieu d'affaires de la zone euro. Cependant, leur description repose moins sur des extraits d'entretiens et des éléments d'ethnographie des pratiques que sur une perspective plus quantitative et plus formaliste. Et ils 
s'en expliquent ainsi en proposant deux étapes : «l'analyse de réseau intervient en première instance pour saisir le degré de proximité des acteurs transnationaux : forment-ils un espace dense d'interconnaissances ou sont-ils isolés les uns des autres œuvrant seulement à mettre en relation des entreprises deux à deux ? ». Cela permet de repérer les interlockers transnationaux, ou acteurclefs. Ces acteurs-clefs sont ensuite étudiés de manière réticulaire et prosopographique afin d'en mieux saisir la cohésion, les similitudes et les différences. En effet, l'analyse prosopographique permet de mesurer les points de rapprochement entre types de carrière et les points d'éloignements liés aux tropismes nationaux des formations, donc de la reproduction des élites. Enfin, en rapprochant les deux modalités, ceci permet d'interroger le poids des dirigeants issus de la finance au sein de ce petit groupe et de déterminer si le poids des financiers au sein de l'économie est ou non disproportionné. La force de cette approche tient à sa capacité de reconstituer les trajectoires professionnelles des acteurs clefs, puis à préciser les profils des différents parcours et à voir à quels types de secteurs ils appartiennent.

Dans un style assez différent, Grégory Daho présente une analyse de la socialisation entre groupes professionnels de la politique étrangère en s'interrogeant sur l'institutionnalisation des activités civilo-militaires françaises en ex-Yougoslavie. Il s'agit pour lui d'étudier les relations entre militaires, diplomates et industriels en observant l'évolution des formes de socialisation, c'està-dire des processus d'intériorisation de normes et de références et d'assimilation entre groupes professionnels, afin de souligner les dynamiques intersectorielles qui les caractérisent. Il en résulte une étude historique des transformations des années 1990 et 2000 qui fait émerger « des officiers des forces spéciales, ni tout à fait militaires, ni espions, ni industriels qui forment une nébuleuse fonctionnant discrètement par cooptation de réseaux et disposant de capacités de reconversion certaines. Des officiers généraux héritiers de l'armée d'Afrique aux parcours sans tâche qui refusent de se contenter de l'immobilisme de la non-guerre nucléaire, qui trustent les positions d'autorité au sein d'une armée réformée et deviennent les cadres du maintien de la paix. Des urgentistes du MAE, plus “opérationnels” que “diplomates”, militants socialistes tournés vers l'univers des ONG, qui se protègent mutuellement en s'aménageant, dans la difficulté, un "espace de liberté” au sein de leur institution ». Ceci permet, en comprenant les jeux intersectoriels, de se mettre à distance de thèses qui insistent bien trop sur l'intentionnalité stratégique des décideurs ou, à l'inverse, sur les pures logiques d'adaptation.

Enfin, en dehors du cadre de cette table-ronde de l'AFSP, nous présentons une traduction en Français de l'article publié dans la revue IPS par un collectif de chercheurs (Zygmunt Bauman, Didier Bigo, Paulo Esteves, Elspeth Guild, Vivienne Jabri, David Lyon, R. B. J. Walker) qui s'était mobilisé pour repenser l'impact de la surveillance après les révélations d'Edward Snowden, en 
analysant leurs conséquences sur la sécurité nationale, les droits de l'homme, la démocratie et les formes de subjectivité et d'obéissance. Le texte revient sur les principales informations que Snowden a données concernant les pratiques de surveillance de la NSA américaine et des autres services SIGINT qui ont collaboré avec elle, ainsi que sur l'étendue et le caractère intrusif de cette surveillance. Longtemps niées, les pratiques d'échange d'informations «sensibles » entre services de renseignement nous donnent sans doute à lire une transformation de la raison d'État et de son rapport au secret, à la vie privée et à l'espace démocratique. La dimension intrusive et de grande échelle de la surveillance semble être la conjonction de trois processus qui vont s'articuler : la transnationalisation, la numérisation et la privatisation, conjonction qui remet en cause les relations entre les professionnels nationaux de la politique et les guildes transnationales de renseignement qui tentent d'avoir un monopole sur les informations « sensibles». Ce texte ouvre la question des choix qui s'ouvrent à nous face à la surveillance : la résignation, la perplexité ou/et la résistance? 